\title{
The Minimal Supersymmetric Standard Model and Precision of $W$-Boson Mass and Top Quark Mass
}

\author{
Kyungsik Kang and Sin Kyu Kang \\ Department of Physics, Brown University, Providence, RI 02912, USA
}

\begin{abstract}
We argue that the present value and accuracy of $M_{W}$ and $m_{t}$ measurements tend to favor the MSSM over the SM. By speculating that a precision of the order $40 \mathrm{MeV}$ and $3 \mathrm{GeV}$ respectively for $M_{W}$ and $m_{t}$ will be achieved at LEP2 and Tevatron, we show that the prospect for the MSSM will be further enhanced as long as the central values of $M_{W}$ and $m_{t}$ do not decrease below the present values. In addition we discuss how this scenario can constrain the Higgs boson mass and distinguish the Higgs boson of the MSSM type from that of the SM.
\end{abstract}


Recent LEP measurements [1:2] have improved so precise that LEP's sensitivity can even detect the passing of TGV train. The $W$-boson mass measured at LEP2, when combined with those at the Tevatron, enables us to narrow $M_{W}$ within $\pm 0.08 \mathrm{GeV}$ [2], while the top quark mass has been measured also with a significantly smaller error $\pm 5.5 \mathrm{GeV}$ [3]. These experimental advances should enable us to examine the effect of the higher order radiative corrections more closely and also the existence of the Higgs boson for which we do not have direct evidence yet. Even the apparent deviation of some of the electroweak parameters such as $R_{b}, R_{c}$ and $A_{L R}(\mathrm{SLD})$ from the standard model (SM) predictions [四], which persisted for several years and caused many theorists [5] to interpret these anomalies as a possible signal of new physics beyond the SM, seems to have been mostly washed away from the very recent precision data from LEP and SLD, thus making the SM more appealing. Although the measurements of most electroweak parameters may appear to be consistent with the SM prediction, we would like to point out in this Letter that the present value and accuracy of $M_{W}$ and $m_{t}$ measurements [2,3] tend to support the MSSM rather than the SM. Strictly speaking, the precision tests until now have resulted only a consistent correlations among the relatively less accurate parameters $M_{W}$ and $m_{t}$ and the unknown parameter $m_{H}$ within the framework of the SM. As we discussed in Ref. [7], because of the strong correlation between $M_{W}$ and $m_{t}$ for a wide range of $m_{H}$, the future precision measurements of $M_{W}$ amd $m_{t}$ beyond the current experimental accuracy would provide a decisive and perhaps the only crucial test for or against the SM and give a profound implication for the origin of the Higgs boson as well as to the Higgs boson searches [8]. In this respect, the determinations of $M_{W}$ and $m_{t}$ from $W^{+} W^{-}$[1,9] and future $t \bar{t}$ threshold measurements [10] could also play an important role on the future precision test of the SM and on the determination of the Higgs boson mass, because such measurements can severely reduce the background and systematic uncertainties. A precision of order $40 \mathrm{MeV}$ for $M_{W}$ and $3 \mathrm{GeV}$ for $m_{t}$ could perhaps be achieved at the LEP2 as well as at the Tevatron [11,12] soon.

In this Letter, we would like to discuss how such precise determinations of $M_{W}$ and $m_{t}$ could serve to test the SM and constrain the indirect bound of the Higgs boson mass. In 
particular, we would like to point out that the current data tends to favor already the MSSM bounds over the SM type. We will also examine how the future determinations of $M_{W}$ and $m_{t}$ with further improved accuracy can provide a decisive clue on the evidence of a new physics beyond the SM, such as supersymmetry (SUSY).

As shown in Table I, the precision of $M_{W}$ and $m_{t}$ measurements has been steadily increased during the past years. While the central value of $m_{t}$ is converging to around 175 $\mathrm{GeV}$, that of $M_{W}$ has been continuously increasing with improved accuracy. As will be shown later, the increase of the central value of $M_{W}$ accompanied by the decrease of experimental uncertainty is potentially interesting since it might at last lead to an evidence of new physics beyond the SM. In Fig.1, we present the correlation between $M_{W}$ and $m_{t}$ in the SM (solid lines) and the MSSM (dashed lines). Also shown in Fig.1 is the least-fit solutions $(-\diamond-)$ of the global fits to the SM for $m_{H}=100-1000 \mathrm{GeV}$. The prediction of $M_{W}$ versus $m_{t}$ is achieved with $\alpha^{-1}\left(M_{Z}\right)=128.896 \pm 0.09$ [13] and $\alpha_{s}\left(M_{Z}\right)=0.118 \pm 0.003$ [14] from treating the relation of the radiative corrections self-consistently, following the scheme described in Ref. [7]. The errors in $\alpha^{-1}\left(M_{Z}\right)$ and in $\alpha_{s}\left(M_{Z}\right)$ alone yield uncertainties on $M_{W}$ of order 20 $\mathrm{MeV}$ and $2 \mathrm{MeV}$, respectively. In Fig.1, the band of SM prediction is obtained by taking the range of $m_{H}$ to be $70 \mathrm{GeV} \lesssim m_{H} \lesssim 1 \mathrm{TeV}$, where we use the current experimental lower limit of $m_{H}$ [2]. On the other hand, the lower bound on $m_{H}$ can theoretically be determined from the vacuum stability condition within the context of the SM [15]. The lower bound on $m_{H}$ from the vacuum stability condition depends on $m_{t}$ and new physics scale $\Lambda$ beyond which the SM is no longer valid. This lower bound on $m_{H}$ increases with $m_{t}$ and decreases as $\Lambda$ is increased. For the numerical values, we use the recent fit [16]

$$
m_{H}(\mathrm{GeV})>133+1.92\left(m_{t}(\mathrm{GeV})-175\right)-4.28 \frac{\alpha_{s}\left(M_{Z}\right)-0.12}{0.006}
$$

for $\Lambda=10^{19} \mathrm{GeV}$. We note that the vacuum stability lower bound on $m_{H}$ becomes higher than the current experimental lower limit for $m_{t} \gtrsim 145 \mathrm{GeV}$. Thus we take experimental bound in the region $m_{t} \lesssim 145 \mathrm{GeV}$ as the lower limit of $m_{H}$ and the vacuum stability lower bound for $m_{t} \gtrsim 145 \mathrm{GeV}$ in Fig.1. The upper line of the SM band corresponds to 
these lower bounds on $m_{H}$ in two regions of $m_{t}$. Thus, in the higher region of $m_{t}$, the upper limit of SM prediction for $M_{W}$ is somewhat lower than the one used by others in the literature [12,17]. The lower line of the SM prediction corresponds to $m_{H}=1 \mathrm{TeV}$ as usual. The MSSM bounds have been calculated by varying the SUSY parameter so that they are consistent with current experimental results of the non-observation of Higgs and SUSY particles at LEP2 [12,17,18. In order to see the implication of the present measurements of $M_{W}$ and $m_{t}$, we also present the current experimental results [2,3], $M_{W}=80.43 \pm 0.08$ $\mathrm{GeV}$ and $m_{t}=175.6 \pm 5.5 \mathrm{GeV}$ in this figure. The best global fit solutions give $M_{W}=$ $80.331 \pm 0.024$ (due to $\left.m_{H}\right) \pm 0.020$ (due to $\alpha^{\prime} s$ ) GeV, somewhat lower than the current world average of $M_{W}$. Although the combined central point of the set $\left(M_{W}, m_{t}\right)$ tends to prefer the MSSM, both the SM and the MSSM may actually be consistent with the present accuracy of $M_{W}$ and $m_{t}$ in view of the uncertainties resulting from those in other input parameters such as $\alpha^{-1}\left(M_{Z}\right), \alpha_{s}\left(M_{Z}\right)$, etc. as mentioned above.

From Fig.2, we see that the Higgs boson mass can be constrained by the present experimental values of $M_{W}$ and $m_{t}$ by $m_{H} \lesssim 370 \mathrm{GeV}$ in general. This is a remarkable improvement in the situation compared to the state of the art in 1995 when we suggested to study $M_{W}$ vs. $m_{H}$ correlation for the first time in Ref. [7]. The central values of $M_{W}$ and $m_{t}$ allow $m_{H} \simeq 67 \mathrm{GeV}$ but with an uncertainty $\Delta m_{H}$ of the order $130 \mathrm{GeV}$ due to the experimental uncertainties $\Delta M_{W}=80 \mathrm{MeV}$ and $\Delta m_{t}=5.5 \mathrm{GeV}$. This is consistent with the recent indirect determination of $m_{H}=121_{-68}^{+119} \mathrm{GeV}$ based on the global fit to the most recent electroweak data 12 .

Now, let us speculate how the future measurement of $M_{W}$ and $m_{t}$ with improved accuracy can provide a decisive test for the SM: let us assume that the central values of $M_{W}$ and $m_{t}$ would not decrease from the current values and that the precision of $M_{W}$ and $m_{t}$ would be

\footnotetext{
${ }^{1}$ Rerun of our ZFITTER program [19] following the scheme in Ref. [0] has reproduced the same results.
} 
improved to the order of $40 \mathrm{MeV}$ and $3 \mathrm{GeV}$ respectively, which will be achieved at the LEP 2 and the Tevatron in the foreseeable future [11]. As one can see from Fig.1, this scenario seems to disfavor the SM compared to the MSSM. If the increasing trend of the central value of $M_{W}$ is to continue above the present one, the precision measurement of $M_{W}$ and $m_{t}$ would provide more distinctive evidence for new physics beyond the SM, in particular, in favor of the MSSM. Thus, as long as the central value of $M_{W}$ is determined to increase above the present value, the future precise measurements of $M_{W}$ and $m_{t}$ could serve a useful window to witness the cracks in the SM and to look for the evidence of new physics. On the other hand, as can be seen from Fig.3, the speculation to have $M_{W}=80.43 \pm 0.04$ $\mathrm{GeV}$ and $m_{t}=175.6 \pm 3 \mathrm{GeV}$ leads to $m_{H} \lesssim 180 \mathrm{GeV}$, in the framework of the SM, with an uncertainty $\Delta m_{H}$ of the order $50 \mathrm{GeV}$ due to the uncertainties $\Delta M_{W}=40 \mathrm{MeV}$ and $\Delta m_{t}=3 \mathrm{GeV}$. However, as shown in Fig.1, this scenario seems to prefer the MSSM even better. As is well known, in the MSSM, the intrinsic upper bound on the lightest Higgs boson mass can be obtained by assuming that SUSY effects are decoupled and the lightest Higgs boson is equivalent to the SM Higgs boson below SUSY breaking scale [16,20]. In the considered range of $m_{t}$, this upper bound turns out to be $m_{H} \lesssim 130 \mathrm{GeV}$ [16,20] which is to be discriminated from the lower bound on the SM Higgs boson mass ( $\gtrsim 133 \mathrm{GeV})$. Thus, if the Higgs boson will be discovered in the range $m_{H} \lesssim 130 \mathrm{GeV}$ and $M_{W}$ and $m_{t}$ will be measured with the above speculated values and accuracy, this will constitute a definite signal in favor of the MSSM.

We note that the upper limit of the SM prediction shown in Fig.1 corresponds to the central values of $\alpha^{-1}\left(M_{Z}\right)$ and $\alpha_{s}\left(M_{Z}\right)$. Thus, the upper limit can be increased as much as the lower limit of the MSSM prediction, if the errors in $\alpha^{-1}\left(M_{Z}\right)$ and $\alpha_{s}\left(M_{Z}\right)$ are included. However, if the central value of $M_{W}$ is to increase by $20 \mathrm{MeV}$ from the present value, the MSSM will again be preferred.

In conclusion, we have pointed out that the current value and accuracy of $M_{W}$ and $m_{t}$ measurements tend to favor the MSSM rather than the SM, although most recent elecroweak data may appear to be consistent with the SM prediction. We have shown that our spec- 
ulated values of $M_{W}$ and $m_{t}$ with the precision of order $40 \mathrm{MeV}$ and $3 \mathrm{GeV}$ respectively, which could perhaps be achieved at the LEP2 as well as the Tevatron in the foreseeable future, could prefer the MSSM even better and provide a decisive clue on the evidence of the MSSM when the Higgs boson would be discovered in the range $m_{H} \lesssim 130 \mathrm{GeV}$.

\section{ACKNOWLEDGMENTS}

One of us (S.K.K.) would like to thank the Korea Science and Engineering Foundation for financial support and also the members of the High Energy Theory Group for the warm hospitality extended to him at Brown University. This work was supported in part by the U.S. DOE Contract No. DE-FG02-91ER 40688-Task A. 


\section{REFERENCES}

[1] The LEP Collaborations ALEPH, DELPHI, L3,OPAL, the LEP Electroweak Working Group and the SLD Heavy Flavour Group, Report No. LEPEWWG/97-01, ALEPH 97032 PHYSIC 97-027, DELPHI 97-30 PHYS 683, L3 Note 2071, OPAL Technical Note TN 472, SLD Physics Note 61, 1997.

[2] J. Timmermans, talk presented at the Int. Symposium on Lepton-Photon Interactions ,Hamburg, 1997.

[3] CDF Collaboration, J. Lys, talk presented at ICHEP96, Warsaw, 25-31 July 1996, to appear in the proceedings; DO Collaboration, S. Protopopescu, talk presented at ICHEP96, Warsaw, 25-31 July 1996, to appear in the proceedings.

[4] The LEP Collaborations ALEPH, DELPHI, L3,OPAL, the LEP Electroweak Working Group the SLD Heavy Flavour Group, Report No. CERN-PPE/95-017, CERN-PPE/96017.

[5] For a recent review, see J. Ellis, lectures presented at the Lake Louise Winter Institute Lake Louise, Alberta, Canada, 1997, Report No. CERN-TH/97-131, hep-ph/9707443, and references therein.

[6] H. E. Haber and G. L. Kane, Phys. Rep. 117, 75 (1985); H. P. Nilles, Phys. Rep. 110, 1 (1984).

[7] K. Kang and S. K. Kang, Z. Phys. C 70, 239 (1996); in Proc. Beyond the standard model IV (Lake Tahoe, Dec. 1994), Report No. BROWN-HET-979, hep-ph/9503478.

[8] J. F. Gunion, H. E. Haber, G. L. Kane and S. Dawson, The Higgs Hunter's Guide, Addison-Wesley 1990.

[9] A. Ballestrero et al, in Proc. the Workshop on Physics at LEPQ, eds. G. Altarelli, T. Sjöstrand and F. Zwirner, CERN Yellow Report 96-01. For precision measurements of 
$M_{W}$ and $m_{t}$ at a muon collider, see V. Barger, M. S. Berger, J. F. Gunion and T. Han, Phys. Rev. D 56, 1714 (1997); S. Dawson, Report No. hep-ph/9512260.

[10] For a recent review on the top quark measurements near threshold, see J. H. Kuhn, lectures presented at SLAC Summer Institute, Stanford, CA, 1995, Report No. TTP96-18; see also S. Dawson [9].

[11] F. Merritt, H. Montgomery, A. Sirlin and M. Swartz, Precision Tests of Electroweak Physics, report of the DPF Committe on Long Term Planning, 1994.

[12] U. Baur, M. Demarteau and Working Group Meambers, to appear in Proc. of the 1996 DPF/DPB Summer Study on New Directions for High-Energy Physics, Snowmass, Colorado, June25-July12, 1996.

[13] S. Eidelman and F. Jegerlehner, Z. Phys. C 67, 585 (1995).

[14] Particle Data Group, R. M. Barnett et al, Phys. Rev. D 54, 1 (1996).

[15] M. Sher, Phys. Rep 179, 273 (1989); G. Altarelli and G. Isidori, Phys. Lett. B 337, 141 (1994); J. A. Casas, J. R. Espinosa and M. Quiros, Phys. Lett. B 342, 171 (1995); Phys. Lett. B 382, 374 (1996).

[16] M. Quiros, to appear in Perspectives on Higgs Physics II, Ed. G. L. Kane, World Scientific, Singapore, Report No. hep-ph/9703412.

[17] W. Hollik, Plenary talk and Theoretical Summary of the Working Group on Electroweak Interations XVI Int. Workshop on Weak Interactions and Neutrinos (WIN '97) Capri, Italy, June 22-28, 1997, Report No. hep-ph/9708339; R. Clare, talk presented at 1997 Aspen Winter Conference on Particle Physics, 23 Jan 1997.

[18] P. H. Chankowski et al. , Nucl. Phys. B417, 101 (1994); D. Garcia and J. Sola, Mod. Phys. Lett. A9, 211 (1994).

[19] D.Bardin et al, Report No. hep-ph/9412201. 
[20] M. Carena, J.R. Espinosa, M. Quiros and C.E.M.Wagner, Phys. Lett. B355, 209 (1995); M. Carena, M. Quiros and C.E.M Wagner, Nucl. Phys. B461, 407 (1996); H.E. Haber, R. Hempfling and A. H. Hoang, Report No. CERN-TH/95-216, hep-ph/9609331. And references therein.

[21] The LEP Collaborations ALEPH, DELPHI, L3,OPAL, the LEP Electroweak Working Group and the SLD Heavy Flavour Group, Report No. CERN-PPE/96-183.

[22] The LEP Collaborations ALEPH, DELPHI, L3,OPAL, the LEP Electroweak Working Group, Report No. CERN-PPE/95-172.

[23] The LEP Collaborations ALEPH, DELPHI, L3,OPAL, the LEP Electroweak Working Group, Report No. CERN-PPE/94-187. 


\section{TABLES}

TABLE I. The evolution of $M_{W}$ and $m_{t}$ measurements.

\begin{tabular}{lcc}
\hline \hline & $M_{W}(\mathrm{GeV})$ & $m_{t}(\mathrm{GeV})$ \\
\hline 1997 (Summer) [2] & $80.43 \pm 0.08$ & $175.6 \pm 5.5$ \\
1997 (January) [1] & $80.37 \pm 0.08$ & $175.6 \pm 5.5$ \\
1996 (Summer) [21 & $80.356 \pm 0.125$ & $175 \pm 6$ \\
1995 (Summer) [22 & $80.26 \pm 0.16$ & $180 \pm 13$ \\
1994 (Summer) 23] & $80.23 \pm 0.18$ & $174 \pm 10_{-12}^{+18}(\mathrm{CDF})$ \\
\hline \hline
\end{tabular}




\section{FIGURES}

FIG. 1. $M_{W}$ versus $m_{t}$ in the SM (solid lines) and the MSSM (dashed lines). The current experimental results, $M_{W}=80.43 \pm 0.08 \mathrm{GeV}$ and $m_{t}=175.6 \pm 5.5 \mathrm{GeV}$ are presented and the speculation to improve the errors by half so that we have $M_{W}=80.43 \pm 0.04 \mathrm{GeV}$ and $m_{t}=175.6 \pm 3.0 \mathrm{GeV}$ are also indicated by the cross points $(\mathrm{xxx})$. The cases of the minimal $\chi^{2}$-fit from the global fits to the recent LEP data are shown by $\diamond$.

FIG. 2. $M_{W}$ versus $m_{H}$ for $m_{t}=175.6 \pm 5.5 \mathrm{GeV}$. The current experimental measurement of $M_{W}$ is shown by dashed lines. The vertical dotted line corresponds to the experimental lower limit of the Higgs boson mass $\left(m_{H}=70 \mathrm{GeV}\right)$.

FIG. 3. The same as Fig.2 but for $m_{t}=175.6 \pm 3.0 \mathrm{GeV}$ and $M_{W}=80.43 \pm 0.04 \mathrm{GeV}$. 
Figure 2

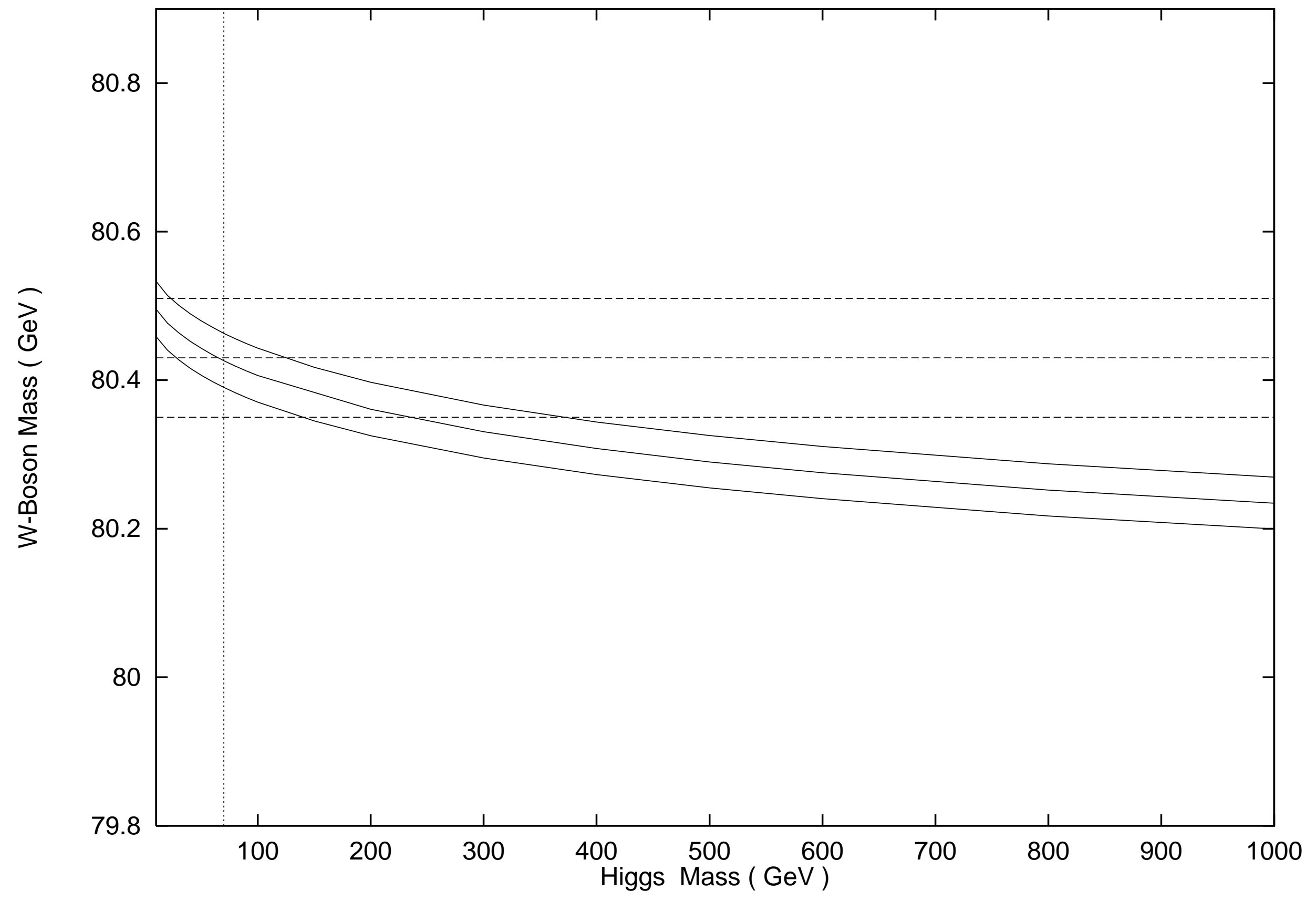


Figure 3

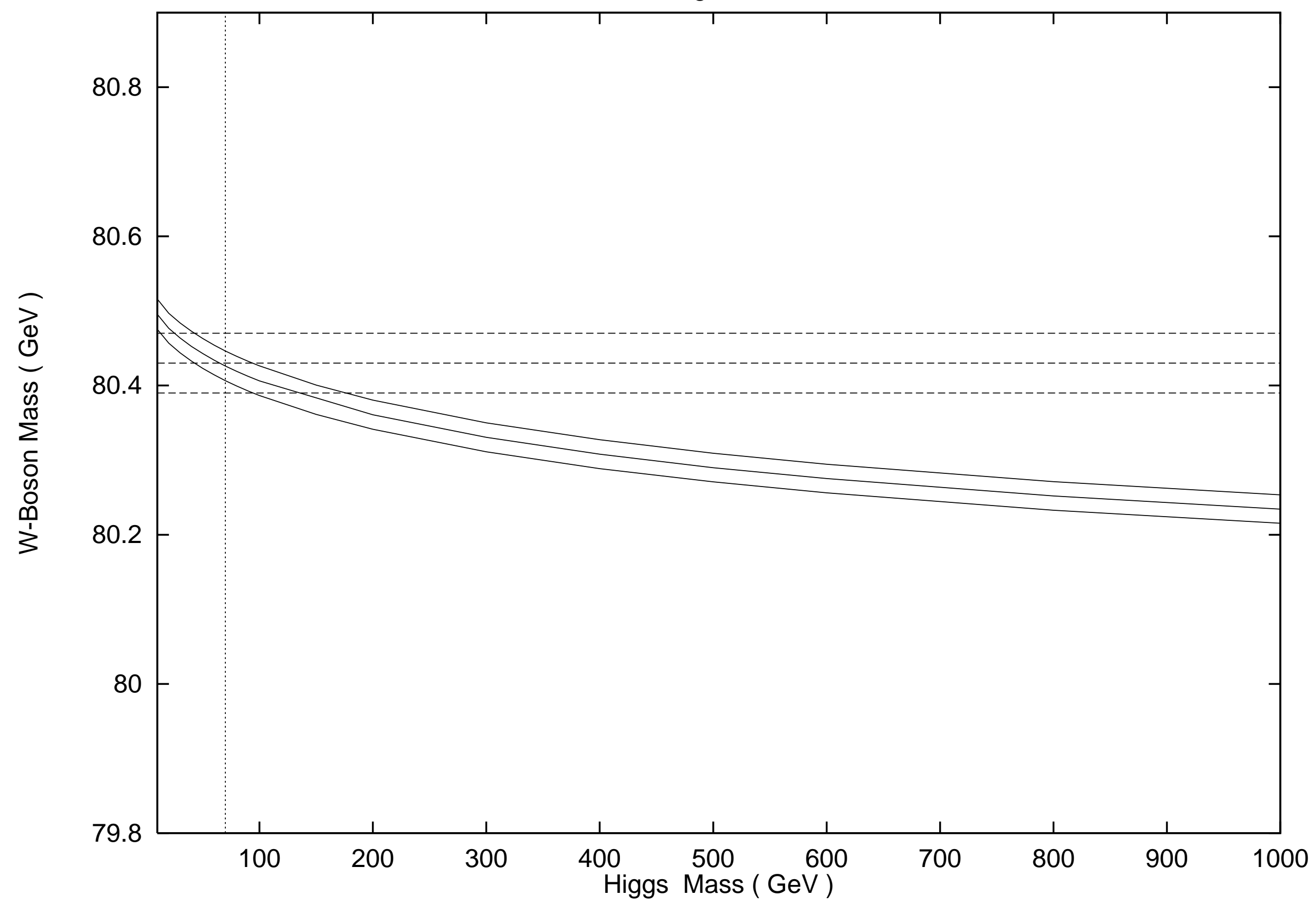


Figure 1

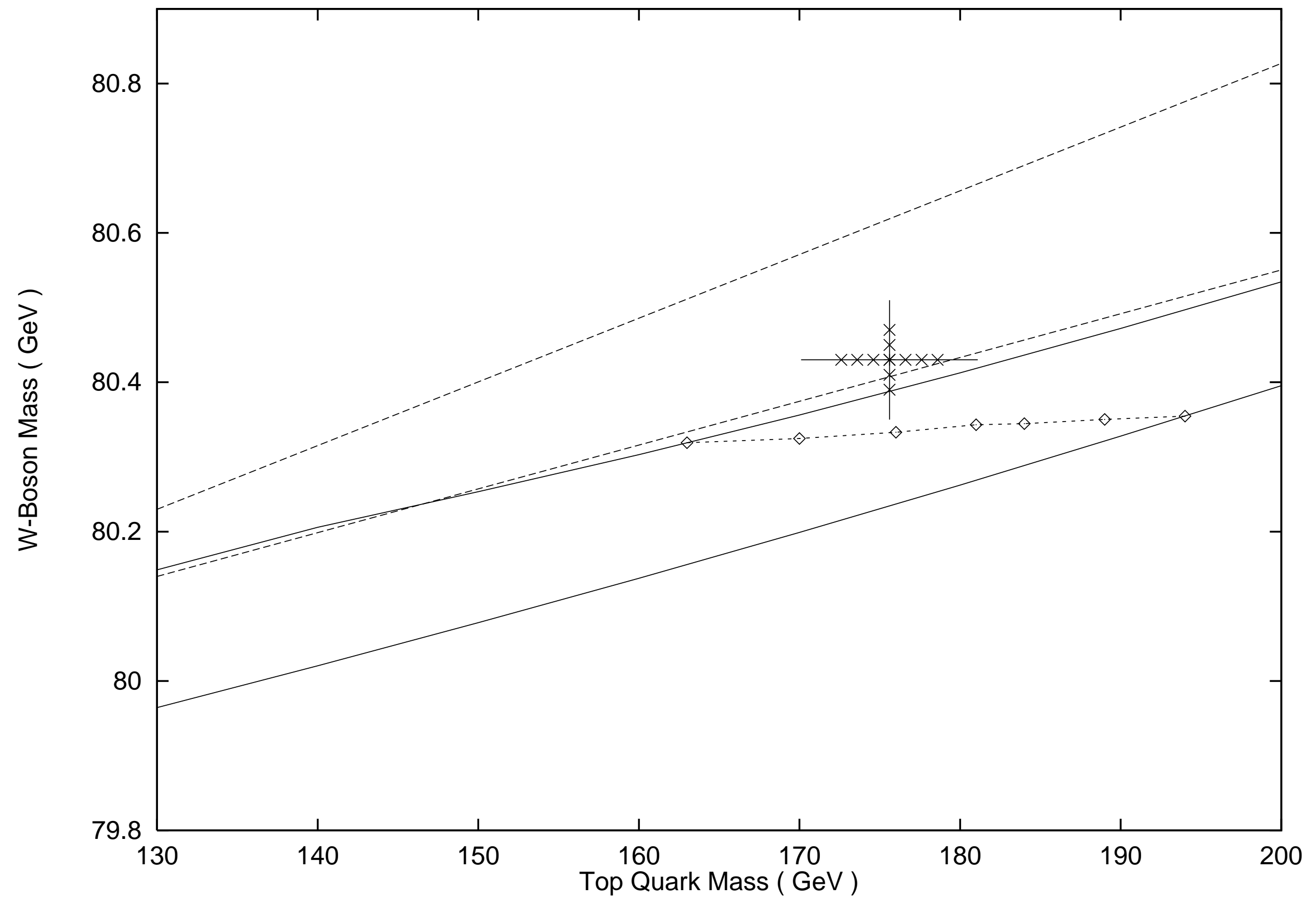

